The Evolution of Language, Proceedings of the

6th International Conference (EVOLANG6),

A. Cangelosi et al. (Eds.) pp. 415-416, World Scientific (2006)

\title{
RECONSIDERING KIRBY'S COMPOSITIONALITY MODEL TOWARD MODELLING GRAMMATICALISATION
}

\author{
TAKASHI HASHIMOTO \& MASAYA NAKATSUKA \\ School of Knowledge Science, \\ Japan Advanced Institute of Science and Technology (JAIST) \\ 1-1, Nomi, Ishikawa, Japan, 923-1292 \\ \{hash,m-naka\}@jaist.ac.jp
}

Grammaticalisation is a potent candidate to structuralise and complexify human languages in the evolution of language. It is a phenomenon of language change, in which content words such as nouns and verbs change into functional words such as auxiliaries and prepositions. New functional categories, tense, mood, and so forth, can emerge in a language structure through grammaticalisation, then structure and lexicon of a language can become complex and fruitful. It is important to understand the process of and the cognitive ability for grammaticalisation in the context of the origin and the evolution of language. We discuss constructing a computational model for grammaticalisation to achieve this end.

It is assumed that reanalysis and analogy are underlying mechanisms of grammaticalisation (Hopper \& Traugott, 2003). Reanalysis is structural change without observable change in forms. This occurs when a hearer understands a form to have a structure differently from that of a speaker. Analogy is to apply a grammatical rule to forms in which the rule was not applied formerly. These mechanisms postulate a coginitive ability to find analogy among situations and among forms. We call the former "linguistic analogy" and the latter "cognitive analogy".

We thoroughly analysed Kirby's compositionality model (Kirby, 2002), especially the relationship between learning mechanisms in the model and the underlying mechanisms for grammaticalisation from the cognitive viewpoint in order to develop a model of grammaticalisation based on reanalysis and analogy. In this model, a language learner acquiring his own grammar performs three operations to generalise his grammar: chunk, merge and replace (the third one is not named in (Kirby, 2002)). Cognitive analogy is premised in chunking and merging. Reanalysis is realised partly in chunking, since a learner can analyse utterances in different way from a speaker's by chunking operation. The important feature of linguistic analogy is expressed in merging and replacing, for a learner extensively applies a grammatical rule, which was used for only an instance, to all members in a category to which the instance belongs. It was also recognised that these two 
operations were so strong that one instance triggers complete integration of different categories. Consequently, reanalysis and analogy are thought of as being modelled in part in Kirby's model.

Accordingly, it is expected that a phenomenon superficially comparable to grammaticalisation is observed in simulations of the model. The meanings in the model, however, consist of verbs and nouns, no function meaning. Thus, we investigated meaning change in which syntactic category of a word varies with time. Grammaticalisation is a subset of this type of meaning change, since syntactic category of a word changes over time such as from verb to auxiliary and from noun to preposition. In search of such meaning change, we slightly modified the model in order not to converge but to keep changing.

We actually observed phenomena in which a form for a noun was to be used commonly for various verbs in simulations of Kirby's model. They occur through the following process: 1) There are two forms for one noun meaning. 2) Both two happen to appear in an utterance of a speaker. 3) A learner analyses one of them as representing the noun and the other as a part of a form for another meaning. 4) The latter form is to acquire another meaning later. Our scrutiny revealed that a meaning change in which syntactic category of a word was transformed was caused by the deviation of intention between speaker and learner, and the differentiation of word meaning brought by the existence of synonyms. We also found that the replacing operation played an important role in this change process.

We introduce function meanings as an additional argument in predicate logical expressions, which are employed as meaning representations, since the Kirby's original model was not able to express a functional meaning. In this study, we used tense, that is, past, present and future. The change of word meaning over content and function categories, such as from nouns or verbs to tense, was also observed. Accordingly, we confirmed that a slight modification of the Kirby's compositionality model can work as a basic model of grammaticalisation.

Further, in order to equip a meaning space with particular structure, two modifications was brought in. One is to change the criterion to apply the chunking operation. The other is to change the appearance frequency of meanings. Both modifications are concerned with a verb "go" and a tense "future". This premises that the agent has a cognitive disposition to consider, or the world has a physical structure, that actions of going often cause something in future. The effect of these modifications on the phenomena of grammaticalisation will be discussed.

\section{References}

Hopper, P. J., \& Traugott, E. C. (2003). Grammaticalization. Cambridge: Cambridge University Press.

Kirby, S. (2002). Learning, bottlenecks and the evolution of recursive syntax. In T. Briscoe (Ed.), Linguistic evolution through language acquisition (pp. 173-203). Cambridge: Cambridge University Press. 